\title{
European Asbestos Conference 2003
}

\section{O. Hagemeyer}

\author{
European Asbestos Conference 2003
}

Vom 3. bis 6. September 2003 fand die 4. Europäische Asbestkonferenz in Dresden statt. Initiiert durch das Seniour Labour Inspector's Committee (SLIC) und die EU-Kommission sowie in Kooperation mit der Internationalen Arbeitsorganisation (ILO) wurde das Treffen durch den deutschen Länderausschuss für $\mathrm{Ar}$ beitsschutz und Sicherheitstechnik (LASI) und den Hauptverband der gewerblichen Berufsgenossenschaften (HVBG) organisiert. Mehr als 160 Teilnehmer aus 29 Staaten sowie Vertreter der Europäischen Kommission und der Internationalen Arbeitsorganisation (ILO) nahmen an der Konferenz in der BG-Akademie für Arbeit und Gesundheit in Dresden teil.

Thematisch gliederte sich die Veranstaltung in drei Hauptgruppen. In der Einführung gelang es Woitowitz (Deutschland) exzellent, aktuelle arbeitsmedizinische Erkenntnisse von Asbest-assoziierten Erkrankungen den anwesenden Technikern, Wissenschaftlern, Vertretern von Unternehmen und Gewerkschaften und Politikern darzulegen. Insbesondere wies er auf die Erfahrungen aus der ehemaligen DDR und das Auftreten von Krebserkrankungen auch bei alleinigem Einsatz von Chrysotil-Asbest hin. Rantanen (Finnland) stellte technische Aspekte der Verwendung von Asbest sowie Möglichkeiten und Erfahrungen der technischen Prävention vor. Er präsentierte Daten zum Asbestverbrauch in 73 Ländern im Zeitraum von 1985 bis 1992. In 14 Ländern stieg der Verbrauch und in 36 Ländern blieb er in dieser Zeit unverändert hoch, nur in 23 Ländern konnte der Asbestverbrauch gesenkt werden. Insgesamt wurden im Jahre 2000 2,13 Mio. $t$ Asbest produziert, wobei gerade in den weniger entwickelten Staaten der Verbrauch steigt (Tab. 1 u. 2). Aus den von Rantanen präsentierten Zahlen zur Asbestproduktion und zum Asbestverbrauch kann einerseits deutlich ersehen werden, dass die Produktion mit Ausnahme von Kanada weitgehend in Nichtindustriestaaten erfolgt und der Verbrauch überwiegend in den bevölkerungsreichsten Ländern der Erde stattfindet. Ein Problem in den Industrieländern stellen die in Produkten (z. B. Gebäuden) gespeicherten Asbestfasern dar, die entsprechend der Lebensdauer der Produkte noch in Jahrzehnten zu einer gesundheitsgefährdenden Exposition bei der Entsorgung führen können.

Interessant waren die ersten Ergebnisse einer Fragebogenaktion an alle Teilnehmerstaaten (Campbell, England). 28 Antworten belegen übereinstimmend die große Bedeutung der Asbest-assoziierten Gesundheitsprobleme, allerdings sind regelmäßige medizinische Untersuchungen nach beendeter Exposition nicht überall üblich. Aus den Antworten ergibt sich auch, dass im Vordergrund der Empfehlungen die völlige Aufgabe von Asbestproduktion und Verwendung steht. In der EU soll ab 15. April 2006 ein vollständiges Verwendungsverbot von Asbest gelten. Die Auswertung der Angaben zur Häufigkeit von Asbest-verursachten Erkrankungen wurde im Rahmen der Diskussion im Sinne einer unzureichenden statistischen Erfassung der Erkrankungen auch in Ländern der EU kritisiert. Darüber hinaus ergaben sich Hinweise, dass in einigen Ländern das Asbestproblem (noch) nicht erkannt wird, in anderen die Zusammenarbeit zwischen Regierung und Arbeitsschutzbehörden nicht optimal funktioniert oder die Vorschriften optimiert werden könnten.

Diese Auswertung leitete in den zweiten Teil über, in dem Länderberichte präsentiert wurden. Die Situation in den EU-Ländern wurde anhand von Berichten aus Spanien, Frankreich, Schweden, Deutschland, Großbritannien, den Niederlanden und Griechenland dargestellt. In allen Ländern sind umfangreiche Maßnahmen zum Schutz der Arbeitnehmer implementiert. Medizinische Untersuchungen zur Sekundärprävention nach Aufgabe der Asbest-belasteten Arbeiten werden nur in wenigen Ländern durchgeführt (z.B. Norwegen, Deutschland), in allen Ländern werden

Institutsangaben

Hauptverband der gewerblichen Berufsgenossenschaften, Sankt Augustin

Korrespondenzadresse

Dr. med. Olaf Hagemeyer · Hauptverband der gewerblichen Berufsgenossenschaften •

Alte Heerstr. $111 \cdot 53754$ Sankt Augustin

Bibliografie

Pneumologie 2004; 58: 50-51 @ Georg Thieme Verlag Stuttgart · New York ·

ISSN 0934-8387 · DOI 10.1055/s-2003-812446 
Asbest-verursachte Erkrankungen aber als Berufskrankheit entschädigt.

In den Beitrittsländern Polen, Bulgarien und Lettland werden derzeit rechtliche Regelungen getroffen, jedoch ist noch in unterschiedlichem Ausmaß von einer bestehenden Asbestexposition auszugehen. Erfahrungen aus diesen Ländern weisen auf eine weit verbreitete Unkenntnis der Gesundheitsgefahren bei Unternehmern u. Mitarb. hin und belegen Schulungsbedarf. Ein generelles Problem in allen Staaten ist die Erfassung und Betreuung von Beschäftigten in Kleinbetrieben und von Selbständigen.

Berichte außerhalb Europas kamen aus Japan, Brasilien und Thailand. Nach Japan wurden 1974 etwa 350000 t Asbest importiert. Zwischen 1978 und 2003 wurden als Berufskrankheit anerkannt und entschädigt 235 Lungenkrebs- und 230 Mesotheliomfälle (zum Vergleich: in Deutschland wurden zwischen 1978 und 2000 anerkannt 6985 Lungenkrebs- und 6626 Mesotheliomerkrankungen). Die Zahl der Mesotheliomerkrankungen gab der Referent andererseits mit 414 im Jahr 2001 an. Ergänzend zum Verbot anderer Asbestarten erwägt die japanische Regierung jetzt auch ein Verbot von Chrysotil-Asbest. Während sich Japan in der Entwicklung auf europäisch vergleichbare Standards befindet, konnten die Berichterstatter aus Brasilien und Thailand dieses nicht zeigen. Das Schutzsystem ist hier allenfalls rudimentär, wenngleich auch Brasilien die ILO-Konvention 162 „Sicherheit bei der Verwendung von Asbest" ratifiziert hat. Aktive Maßnahmen zur Förderung des Schutzes vor Asbestgefahren werden mit allen Mitteln (bis hin zu Bedrohungen des persönlichen Lebens) bekämpft.

Im dritten Teil bereiteten fünf Workshops über Identifizierung von Asbest bei Gebrauch und Instandhaltung, gute Praktiken zur Beseitigung von Spritzasbest, über Gesetzgebung, Kontrolle und Vollzug, arbeitsmedizinische Betreuung und Epidemiologie sowie zur Aus- und Fortbildung die Erarbeitung der „Dresdner Erklärung zum Schutz der Arbeitnehmer vor Asbest“ vor. Der Berichterstatter nahm am Workshop über arbeitsmedizinische Betreuung teil. Als wesentliches Ergebnis wurde die Forderung nach einer internationalen Konferenz zur Standardisierung der medizinischen Untersuchungen nach beendeter Asbestexposition von allen Teilnehmern begrüßt.

Eine Posterausstellung vermittelte ergänzende Aspekte, wobei diejenigen zur Früherkennung von Tumoren (mit Low-Dose-Spiral-CT und Sputumanalyse bei Hochexponierten) und zur arbeitsmedizinischen Betreuung nach Beendigung der Asbestexposition dominierten.

Adressaten der „Dresdner Erklärung zum Schutz der Arbeitnehmer vor Asbest“" sind die Europäische Kommission, die EU-Staa-
Tab. 1 Asbestproduktion und Asbestverbrauch in 2000 (Angaben nach: Rantanen, Finnland)

\begin{tabular}{|ll|}
\hline Staat & Produktion (t) \\
\hline Russland & 700000 \\
\hline China & 450000 \\
\hline Kanada & 335000 \\
\hline Kasachstan & 180000 \\
\hline Brasilien & 170000 \\
\hline Simbabwe & 130000 \\
\hline Swasiland & 25000 \\
\hline Indien & 23000 \\
\hline Südafrika & 20000 \\
\hline Japan & 18000 \\
\hline
\end{tabular}

Tab. 2 Asbestverbrauch in 2000. Weitere Staaten mit einem Verbrauch von mehr als 10000 t pro Jahr sind: Süd-Korea, Mexiko, Belorussland, Iran, Türkei, Malaysia, Kirgisien, Spanien, USA, Süd-Afrika, Nigeria, Kolumbien, Rumänien und Simbabwe (Angaben nach: Rantanen, Finnland)

\begin{tabular}{ll}
\hline Staat & Verbrauch $(\boldsymbol{t})$ \\
\hline Russland & 447000 \\
\hline China & 410000 \\
\hline Brasilien & 182000 \\
\hline Indien & 125000 \\
\hline Thailand & 121000 \\
\hline Japan & 98600 \\
\hline Vietnam & 62000 \\
\hline Ukraine & 60000 \\
\hline Indonesien & 54900 \\
\hline Kasachstan & 32400 \\
\hline
\end{tabular}

ten und assoziierten Staaten, die Sozialpartner und die ILO. Diese werden aufgefordert u.a. den Import Asbest-verunreinigter Produkte in und den Export von Asbest-verseuchten Abfalls aus der EU zu verhindern, zentrale Register der Exponierten zu erstellen, medizinische Vorsorgemaßnahmen nach beendeter Exposition (Sekundärprävention) zu verbessern und die Asbest-verursachten Erkrankungen in der Liste der Berufskrankheiten besser zu berücksichtigen.

Die Europäische Asbestkonferenz 2003 betont als ultimatives Ziel aller Bemühungen die weltweite Einstellung von Asbestproduktion und Asbestverbrauch. 